\title{
Transport mechanism in granular Ni deposited on carbon nanotubes fibers
}

\author{
M. Salvato, ${ }^{*}$ M. Lucci, I. Ottaviani, and M. Cirillo* \\ Dipartimento di Fisica and MINAS Lab, Università di Roma "Tor Vergata," I-00133 Roma, Italy \\ E. Tamburri, S. Orlanducci, and M. L. Terranova \\ Dipartimento di Scienze e Tecnologie Chimiche, MINAS Lab, Università di Roma “Tor Vergata,” I-00133 Roma, Italy \\ M. Notarianni, C. C. Young, N. Behabtu, and M. Pasquali \\ Department of Chemical and Biomolecular Engineering, Department of Chemistry, The Smalley Institute for Nanoscale Science \\ and Technology, MS-362, Rice University, Houston, Texas 77005, USA
}

(Received 25 July 2012; published 12 September 2012)

\begin{abstract}
We investigate the transport properties of granular nickel electrodeposited on carbon nanotube fibers by measuring the electrical resistance and the current voltage characteristics as a function of the temperature. The bare fiber is governed by a three-dimensional variable range hopping transport mechanism, however, a semiconducting to metallic transition is observed after the Ni deposition as a consequence of the evolution from weak to strong coupling between the deposited nickel grains. The experimental results indicate that the charge transport in the Ni-coated fiber develops from hopping governed by the Coulomb blockade in the case of small grains dimensions to a metallic electron phonon interaction mechanism for large grains dimensions. Tunneling enhanced by thermal fluctuation is responsible for the transport in the intermediate conductivity range. The role of the fiber and the effects due to the magnetic nature of the nickel grains are also discussed.
\end{abstract}

DOI: 10.1103/PhysRevB.86.115117

PACS number(s): 73.63.Fg, 61.46.Fg, 73.22.-f, 78.67.Ch

\section{INTRODUCTION}

The transport mechanism in granular systems is governed by several parameters which are related to grain dimensions and density and are affected by temperature, charge effects, as well as external fields. ${ }^{1}$ The regime of granularity is achieved when the conductance of the samples $G=1 / R$, normalized with respect to its quantum value $2 e^{2} / h$ ( $e$ is the electron charge and $h$ is the Planck constant), is less than the normalized conductance $g_{0}$ of the single grain. Moreover, the samples can be distinguished as metallic or insulator depending on whether the value of the normalized conductance $g=G /\left(2 e^{2} / h\right)$ is, respectively, greater or smaller than the critical value $g_{C}=(1 / 6 \pi) \ln \left(E_{C} / \delta\right)$, where $E_{C}=e^{2} / 4 \pi \varepsilon_{0} \varepsilon d$ is the charging energy of a single grain and $\delta=1 / N\left(\varepsilon_{F}\right) V_{0}$ is the separation between the quantum energy levels due to the finite dimension of each grain. ${ }^{2}$ In these expressions, $\varepsilon_{0}$ is the permittivity, $\varepsilon$ is the dielectric constant, $d$ is the grain diameter, $N\left(\varepsilon_{F}\right)$ is the density of the states at the Fermi energy $\varepsilon_{F}$, and $V_{0}$ is the volume of the single grain. The charging energy $E_{c}$ plays the role of a charge blocking potential and the current flows through the granular systems if the external field or temperature effects are strong enough to overcome its value. For low external fields, a tunneling process can be established and the electrical current flows through the granular system by sequential tunneling or cotunneling. ${ }^{3}$ In sequential tunneling each electron hops between two adjacent grains as in a separate quantum event until the opposite electrode is reached; in cotunneling, the electrons tunnel between the two electrodes passing through all the grains in between as in a single tunnel event. Whichever the mechanism responsible for the conduction in the insulating regime, it is well established experimentally that the electrical resistance in granular systems diverges as $T \rightarrow 0$ following the $R=R_{N} e^{\left(T_{0} / T\right)^{1 / 2}}$ law, where $R_{N}$ is the resistance at a given temperature and the value of the parameter $T_{0}$ depends on the theoretical model adopted. ${ }^{1,4,5}$ In the case of cotunneling, ${ }^{3} T_{0}=2.8 E_{c} d / k_{B} \xi$, where $k_{B}$ is the Boltzmann constant and $\xi$ is the electron localization length. In the case of magnetic granular particles, the temperature dependence of the resistance remains unchanged but the parameter $T_{0}$ is enhanced by a factor $(1+\ln 2){ }^{6}$

Besides charging energy, thermal effects also play a role in the charge transport of granular systems. These effects have been studied and corrections to the classical Drude formula of resistivity have been proposed $;{ }^{7}$ the corrections depend on the relation between thermal energy $k_{B} T$ and the quantity $g \delta$. For $k_{B} T>g \delta^{7,8}$ the electrons are localized inside a single grain and transport is possible only if strong coupling between the adjacent grains is established: In this case a logarithmic temperature dependence of the conductivity is found. For $k_{B} T<$ $g \delta$ (Ref. 2) the electrons are localized on distances longer than the single grain and charge transport is possible even in the case of weak coupling between the grains and corrections to the conductivity, depending on the system dimensionality, are proposed.

The thermal effects assume particular relevance in the case of large grain dimensions when the capacitance $C_{0}$ between two grains becomes large enough that the charging energy $E_{C}=e^{2} / 2 C_{0}$ is negligible. ${ }^{9}$ Due to thermal fluctuations a random electron motion is established in the conducting region generating a voltage fluctuation across the tunnel junction whose average value is $\left\langle V_{T}^{2}\right\rangle=k_{B} T / C_{0}$. For $e V_{T} \cong E_{C}$ a thermal fluctuation induced tunneling (FIT) mechanism is established and the electrical resistance is given by $R=$ $R_{N} e^{T_{1} /\left(T+T_{2}\right)}$, where $T_{1}$ and $T_{2}$ are two parameters such that $T_{1} / T_{2}=\pi w \sqrt{2 m U_{0}} / 2 \hbar .{ }^{9}$ Here $w$ is the distance between the grains, $U_{0}$ is the energy barrier between them, $m$ is the electron mass, and $\hbar$ is the reduced Planck constant. At even higher 
temperature, thermal activation is achieved as implied by the FIT expression for $T \gg T_{2}$.

Granular systems have recently attracted attention because of their potential versatility in bottom up fabrication technology as well as for the study of transport phenomena related to the coupling between the grains. ${ }^{1}$ Particular interest is devoted to magnetic nanostructures because of their possible use in spintronic nanodevices, magnetic storage data supports, sensors, and actuators. Moreover, in view of the development of hybrid materials and structures, the interaction of different nanomaterials and their transport properties is becoming of great interest for nanotechnology applications. Stimulated by these arguments, we electrodeposited nickel nanoparticles on carbon nanotubes (CNTs) fibers and studied the electrical properties as a function of the grain dimension. CNTs represent one of the most studied systems ${ }^{10}$ in the nanotechnology realm and is used in the form of fibers in our experiment as a "substrate." Due to their unique thermal, electrical, and mechanical properties, CNT fibers are promising candidates for the development of hybrid nanotechnology. Their semiconducting character, expressed by a resistivity of the order of few $\mathrm{m} \Omega \mathrm{cm}$, allows both their use as an electrode in an electrolytic cell, and as a substrate for metallic materials. Moreover, because of their uniformity and flexibility, tens of meters of fibers in a unique batch are currently fabricated. Our experimental results provide indications on the transport mechanisms valid for granular systems but also give indications regarding a possible development for metallic or magnetic interconnection nanotechnology.

All the above mentioned parameters relative to granular systems physics can be readily estimated for our samples. The grains dimension in our case ranges between 200 and $45 \mathrm{~nm}$ (as will be detailed in Sec. II below) which gives $E_{c}$ in the (0.7-3.2) $\mathrm{meV}$ interval, assuming $\varepsilon=10^{11}$ for $\mathrm{Ni}$. Moreover, such large grain diameters give a negligibly small value of the quantum energy level separation associated to each grain. Considering a density of states at Fermi level $N\left(\varepsilon_{F}\right) \sim 10^{8} \mathrm{eV}^{-1}$ for our grains, ${ }^{12}$ we have $\delta \sim 10^{-9} \mathrm{eV} \ll$ $E_{c}$. As a consequence, the critical conductance $g_{c} \sim 2$ is obtained. Moreover, the order of magnitude of the capacitance $C_{0}$ associated with each grain results to be $8 \times 10^{-17} \mathrm{~F}$ and $V_{T} \sim$ $1 \mathrm{meV} \sim E_{c}$. Therefore, we expect that thermal effects become important at least for the samples with largest grains. Finally, since $k_{B} \mathrm{~T} \gg g \delta$ for all the samples, a logarithmic temperature dependence of the conductivity should be observed for the whole temperature range investigated. ${ }^{2,7,8}$ We performed $R$ vs $T$ and current-voltage ( $I-V)$ measurements on Ni granular samples with different grain dimensions deposited on CNT fibers. From the experimental results we find evidence of a metal-insulating (M-I) transition which is generated both by the increasing of the grain dimensions and temperature effects. Different regimes of conduction are identified and the experimental results are discussed on the basis of the aforementioned models.

The remainder of the paper is structured as follows: The next section describes fabrication and measurement techniques and presents results of the characterization of the samples. Section III discusses the evidence of a metal-insulator transition that occurs as a consequence of grain sizing. Section IV discusses other effects of grain dimensions and Sec. V concludes the paper.

\section{SAMPLES FABRICATION AND CHARACTERIZATION}

Well aligned macroscopic fibers composed of $\mathrm{HiPco}$ single-walled carbon nanotubes (average diameter and length, respectively, of the order of 1 and $500 \mathrm{~nm}$ ) were produced via solution spinning of a CNT liquid crystal following the protocol described elsewhere. ${ }^{13}$ The controlled decoration of the fibers has been realized by electrochemical routes optimized in previous research. ${ }^{14}$ The fibers' diameter is $D=95 \mu \mathrm{m}$, their mechanical strength $\sim 100 \mathrm{MPa}$, and their room-temperature electrical resistivity $\sim 4 \mathrm{~m} \Omega \mathrm{cm}$. These fibers behave as semiconductors at room temperature and below. ${ }^{15}$ The dimensions of the Ni nanoparticles depend on the deposition time. ${ }^{14}$ The measurements shown in this paper are representative of tens of samples obtained electrodepositing $\mathrm{Ni}$ nanoparticles by chronoamperometry at five deposition times (resp. 30, 60, 75, 80, and 90 seconds) and using fibers that were taken from a single spinning drum and hence had properties uniform within a rough $10 \%$ spread. The selected samples are named following the average grains dimension notation (e.g, $45 \mathrm{~nm}$ refers to a sample with an average grain diameter $d=$ $45 \mathrm{~nm}$ ). The structure of the obtained samples was systematically analyzed by scanning electron microscope (SEM).

Figure 1(a) shows four representative images obtained by SEM of the fiber surface covered by $\mathrm{Ni}$ nanoparticles recorded at different deposition times. The presence of the $\mathrm{Ni}$ nanoparticles separated from each other is evident as well as their increasing spatial density with the deposition time. The grain dimensions were estimated by enlarging the SEM images and averaging the grain diameter measured in different zones. The smallest Ni grains which contribute to the electrical transport are obtained after $30 \mathrm{~s}$ of deposition time and have a diameter $d=45 \mathrm{~nm}$. Increasing the deposition time, the grain dimensions increase linearly (and as a consequence their distance decreases). The obtained data are reported in Fig. 1(b) together with the resistance of the samples recorded at $T=5 \mathrm{~K}$. The data yields a possible interpretation of the growth mechanism of the final Ni layer. Increasing the deposition time, the Ni nanoparticles approach each other on the fiber surface. After about $60 \mathrm{~s}$ the distance between the grains is strongly reduced and the electrical resistance drops down towards the lowest value $R=0.26 \Omega$ achieved for the last sample, which appears uniformly covered by $200 \mathrm{~nm} \mathrm{Ni}$ grains as evidenced by the SEM image [inset of Fig. 1(b)].

The transport properties were studied by measuring $I-V$ characteristics and $R$ vs $T$ at temperatures between $T=4 \mathrm{~K}$ and $T=300 \mathrm{~K}$ using a cryocooler. In some cases, temperature as low as $1.5 \mathrm{~K}$ was reached by plunging the samples in a liquid He cryostat and pumping the vapors. Standard four contacts measurements were performed after fixing the samples on an insulating $\mathrm{SiO}_{2}$ substrate previously patterned with four inline gold contacts. The distance between the inner voltage contacts was $l_{V}=2 \mathrm{~mm}$, while the distance between the outer current leads was $l_{I}=6 \mathrm{~mm}$. This configuration yielded a contact free voltage measurement and a uniform current distribution.

Figure 2 shows the resistances normalized with respect to their values $R_{N}$ at $T=290 \mathrm{~K}$ as a function of the temperature for different deposition times. Also shown is the normalized resistance of the bare fiber before the $\mathrm{Ni}$ electrodeposition which indicates its semiconducting character in the whole 


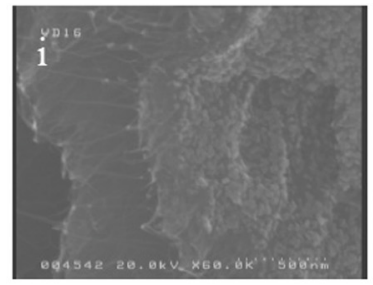

$45 \mathrm{~nm}$

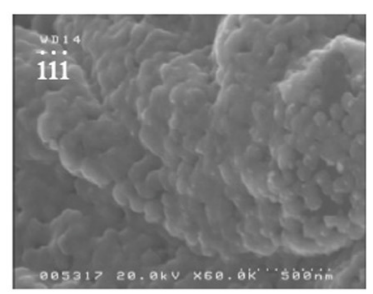

$140 \mathrm{~nm}$

(a)

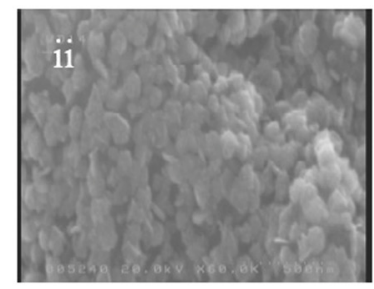

$115 \mathrm{~nm}$

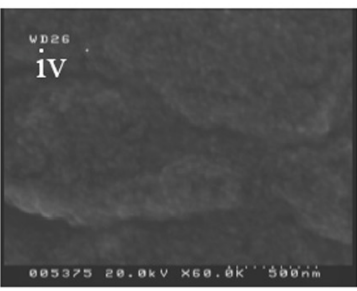

$150 \mathrm{~nm}$

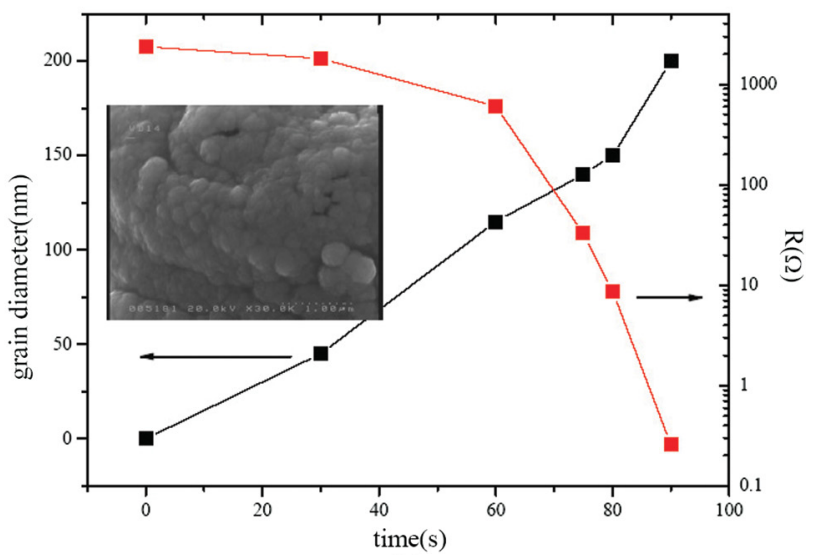

(b)

FIG. 1. (Color online) (a) SEM images of four representative samples with average grain diameter (i) $45 \mathrm{~nm}$, (ii) $115 \mathrm{~nm}$, (iii) $140 \mathrm{~nm}$, and (iv) $150 \mathrm{~nm}$. (b) Grain dimension and electrical resistance of the granular $\mathrm{Ni}$ samples as a function of the electrodeposition time (lines are guide to the eye). Inset: SEM image of the $200 \mathrm{~nm}$ sample surface.

temperature range investigated. This effect has been previously studied $^{16}$ and a three-dimensional variable range hopping (VRH) regime ${ }^{5}$ has been evidenced with a $R$ vs $T$ dependence well fitted by the expression $R=R_{N} e^{\left(T_{M} / T\right)^{1 / 4}}$ in the temperature range $3-50 \mathrm{~K}$. Depositing $\mathrm{Ni}$ nanoparticles on the fiber surface generates an enhancement of their metallic properties. For short deposition times the fibers are still semiconducting with a negative slope in the $R$ vs $T$ curve. This appears clear from the inset of the same figure where the temperature coefficient of the resistance (TCR) given by the expression $\alpha=(1 / R) d R / d T$ is reported. Whereas the semiconducting bare fiber, the 45 and the $115 \mathrm{~nm}$ samples present a negative $\alpha$ value in the whole temperature range, the other curves show an intriguing behavior mainly at low temperature. The $140 \mathrm{~nm}$ sample, for example, although semiconducting, shows a positive value of $\alpha$ below $5 \mathrm{~K}$. On the other hand, the metallic $150 \mathrm{~nm}$ sample presents a clear transition to the semiconducting state below $35 \mathrm{~K}$ and the last deposited $200 \mathrm{~nm}$ sample is metallic in the whole temperature range.

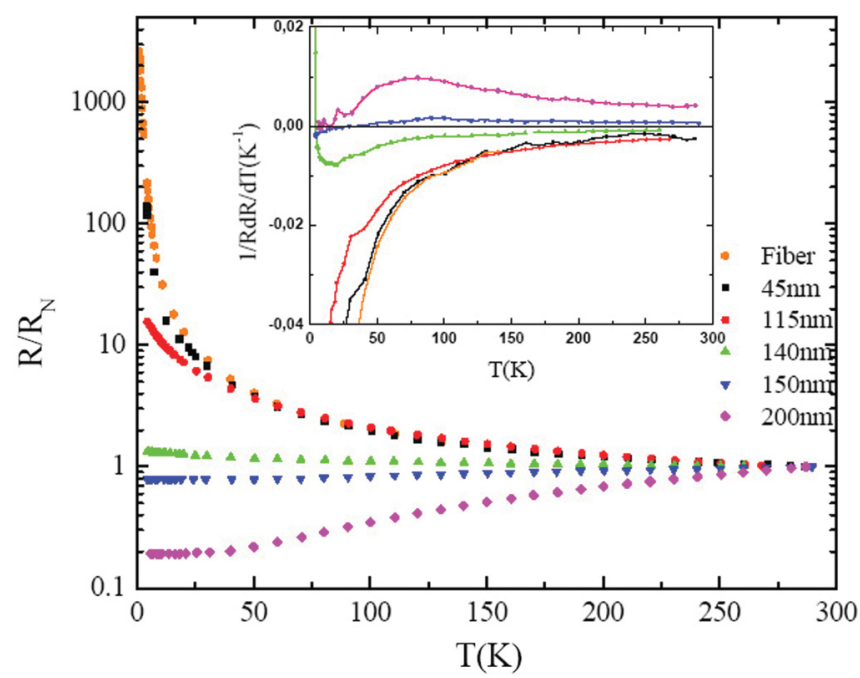

FIG. 2. (Color online) Normalized resistance with respect to its value $R_{N}$ at $T=290 \mathrm{~K}$ as a function of the temperature for all the analyzed samples. Inset: Temperature coefficient of the resistance as a function of the temperature for all the samples.

\section{METAL AND INSULATING REGIME}

The M-I transition observed in Fig. 2 is clearly due to the increasing dimension of the $\mathrm{Ni}$ grains and the consequent reduction of the intergrain distance. In order to give an interpretation of the transport mechanism, the granularity regime should be demonstrated by estimating the conductance $g_{0}$ of the single grain which can be done by the $R$ vs $T$ measurement of the most metallic $200 \mathrm{~nm}$ sample reported in Fig. 3. The resistance of this sample at $T=5 \mathrm{~K}\left(R_{200 \mathrm{~nm}}=0.26 \Omega\right)$ is roughly four orders of magnitude less than the resistance of the bare fiber $\left(R_{F} \approx 700 \Omega\right)$. This decrease implies that most of the bias current flowing through the $200 \mathrm{~nm}$ sample passes inside the Ni layer which is in parallel with the fiber.

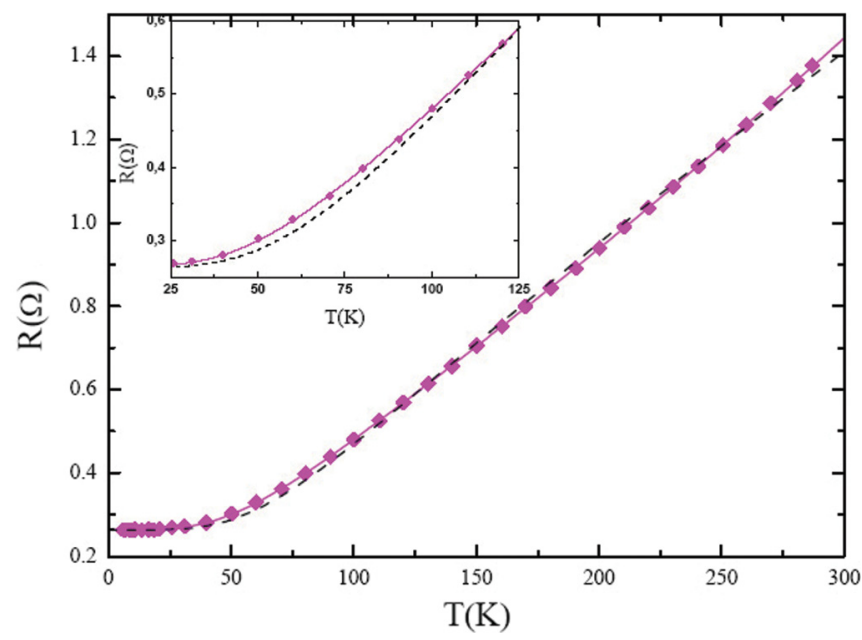

FIG. 3. (Color online) Temperature dependence of the resistance for the $200 \mathrm{~nm}$ sample. The dashed line is a fit to the data following the BG theory for metallic materials where just electron-phonon interactions are considered; the solid line is obtained by the BG theory considering the effects of electron-magnons interactions. Inset: Zoom of the low temperature part of the data shown in the main panel. 
Schematically, this Ni layer can be represented as a compact network of spherical Ni nanoparticles of diameter $d$ arranged in a monolayer on the cylindrical fiber surface consisting of roughly $n_{l}=l_{V} / d=10000$ and $n_{c}=\pi D / d=1570$ grains organized along the fiber length and the circumference, respectively.

Assuming that the grains contribution to the resistance of the $200 \mathrm{~nm}$ sample is that of a network consisting of $n_{l}$ series and $n_{c}$ parallel resistances, we obtain for the resistance of a single grain the expression $R_{0}=\left(n_{c} / n_{l}\right) R_{200 \mathrm{~nm}}=0.0408 \Omega$ at $T=5 \mathrm{~K}$ where $R_{200 \mathrm{~nm}}(T=5 \mathrm{~K})=0.26 \Omega$. This value gives for the normalized conductance for the single grain the value $g_{0}=3 \times 10^{5}$. Since $g_{200 \mathrm{~nm}}=\left(1 / R_{200 \mathrm{~nm}}\right) /\left(2 e^{2} / h\right)=$ $5 \times 10^{4}<g_{0}$ and the $200 \mathrm{~nm}$ sample has the maximum conductance, all the samples show $g<g_{0}$ and therefore all of them can be considered in the granularity regime. The dashed curve in Fig. 3 is the best fit to the data obtained by using the Bloch Gruneisen (BG) $\operatorname{model}^{17}$ for metallic systems where just the intraband electron phonon interactions are considered. The fit is reasonably good confirming that our hypothesis of a uniform monolayer of $\mathrm{Ni}$ grains on the fiber surface for this sample is consistent as also suggested by the SEM image. The solid curve in Fig. 3 instead is the best fit of the experimental data following the same theoretical model but corrected with the term $T^{2}$ due to low temperature electron-magnon interactions. ${ }^{18}$ The solid curve, as emphasized even in the zoom reported in the inset, improves significantly the fit indicating that the Ni layer deposited on the fiber surface consists of a metallic ferromagnetic layer.

In the upper inset of Fig. 4 the $I-V$ characteristics at different temperature are shown for the semiconducting $45 \mathrm{~nm}$ sample. The upward curvature drops as the temperature rises but their shape are reminiscent of that already observed in monolayer of granular systems showing inelastic cotunneling mechanism. ${ }^{3}$ The curves are fit to the data at $T=1.5 \mathrm{~K}$

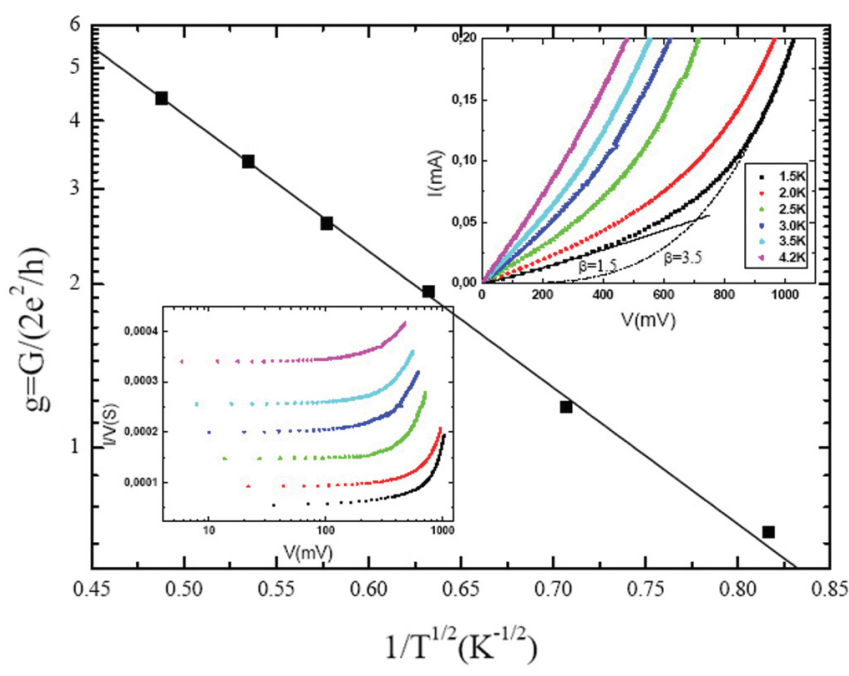

FIG. 4. (Color online) Normalized conductance as a function of $T^{1 / 2}$ for the $45 \mathrm{~nm}$ sample. The lines are fit to the data following the expression reported in the text. Upper inset: $I-V$ curves of the same sample at different temperature. The lines are power law fits to the data $I \propto V^{\beta}$ of the $1.5 \mathrm{~K}$ curve with $\beta=1.5$ (solid line) and $\beta=3.5$ (dashed line). Lower inset: Conductance as a function of the voltage bias at different temperature. obtained by power law dependence $I \propto V^{\beta}$ with $\beta$ between 1.5 and 3.5. Following the cotunneling model, the exponent $\beta$ should be equal to the number of junctions between the electrodes that, in our case, results to be of the order of $10^{4}$. However, as already observed for in plane conductance in granular systems, ${ }^{3}$ a combined mechanism of sequential tunnel and cotunneling is possible. This suggests that charge carriers travel over possible percolative paths joined between them by few tunnel junctions. This is commonly observed in metallic granular systems giving $I-V$ characteristics with an upward curvature which diminishes increasing the temperature. , $^{3,19}$

The conductance vs bias voltage, obtained by the $I-V$ characteristics for the same temperatures, is reported for the same sample in the lower inset of Fig. 4. Here the temperature dependence of the conductance is obtained by extrapolation in the limit of low bias voltage. This, normalized with respect to the quantum value, is reported in the main panel of Fig. 4 in semilogarithmic scale. The lowest value obtained at the lowest temperature $(T=1.5 \mathrm{~K})$ gives $g=0.7<g_{c}$ previously estimated for our grains. The line is a fit to the data obtained by the expression $g \propto e^{\sqrt{T_{0} / T}}$ with $T_{0}=33.4 \mathrm{~K}$. From this value we obtain an electron localization length $\xi=140 \mathrm{~nm}$. These data and fit demonstrate that the sample $45 \mathrm{~nm}$ is in the semiconducting regime where the charge carriers are localized on distances larger than the grain diameter $d$. This means that grain agglomerates form on the fiber consisting of about three Ni grains. Each electron hops between these agglomerates along a distance given by the hopping length ${ }^{5}$ $r=\sqrt{e^{2} \xi / 4 \pi \varepsilon_{0} \varepsilon k_{B} T}=388 \mathrm{~nm}$ calculated at $T=5 \mathrm{~K}$. The correction to these data taking into account magnetic effects of the ferromagnetic grains gives $T_{0}=19.7 \mathrm{~K}, \xi=81 \mathrm{~nm}$, and $r=300 \mathrm{~nm}$ which do not alter the essence of the interpretation of our data.

\section{INTERMEDIATE REGIME AND M-I TRANSITION}

Increasing the grains dimension, a different transport mechanism takes place. This change can be due either to a strong coupling mechanism between the grains or to the fact that the blocking effect of $E_{c}$ is weakened. Strong coupling predicts a logarithmic temperature dependence of the normalized conductance at $T>g \delta / k_{B} \cdot{ }^{7,8}$ In Fig. 5 the $g$ vs $T$ dependence calculated in the same way as in the case of the $45 \mathrm{~nm}$ sample is shown for the 115 and $140 \mathrm{~nm}$ samples. The data are plotted in semilogarithmic scale; in both cases, the logarithmic dependence on temperature is observed only at temperature above 20 and $50 \mathrm{~K}$ for 115 and $140 \mathrm{~nm}$, respectively. Following the aforementioned strong coupling model, ${ }^{2,6,7}$ a logarithmic correction to the temperature should be achieved when $T>g \delta / k_{B}$, which in the case of these samples is at maximum $70 \mathrm{mK}$. This suggests that, besides qualitatively, this model is not appropriate to our system.

The effect of the increasing grains dimension on the Coulomb energy $E_{c}$ gives very interesting results. Because $V_{T}$ also decreases with $d$ but at a rate lower than $E_{c}$, we could expect that thermal effects become relevant. In Fig. 6 the normalized resistances of the 115 and $140 \mathrm{~nm}$ samples are reported as a function of the reciprocal of the temperature. For these samples, $E_{c}$ and $V_{T}$ calculated at $T=5 \mathrm{~K}$ give 


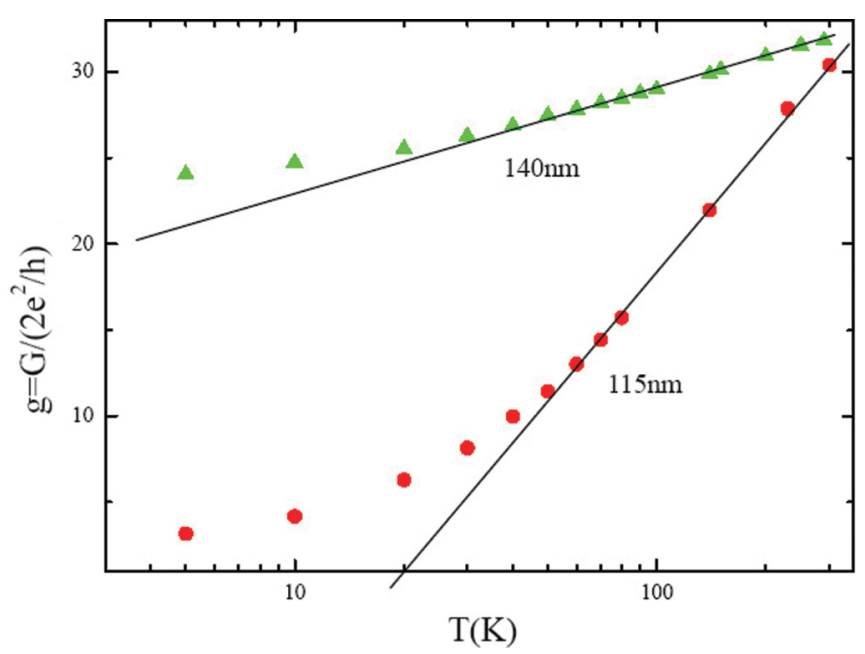

FIG. 5. (Color online) Normalized conductance as a function of the temperature for the 115 and $140 \mathrm{~nm}$ samples. The lines are fit to the data in the high temperature regions following the strong coupling model between the grains.

$1 \mathrm{meV}$ and the FIT model can be acceptable. The curves in Fig. 6 are the fits to the data obtained using the expression of the FIT model with $T_{1}=131.6 \mathrm{~K}$ and $T_{2}=39.7 \mathrm{~K}$ for the $115 \mathrm{~nm}$ and $T_{1}=36.9 \mathrm{~K}$ and $T_{2}=95.9 \mathrm{~K}$ for the $140 \mathrm{~nm}$ sample. The FIT model applies to disordered samples which show a finite resistance in the limit of zero temperature. This model assumes that the otherwise localized carriers are free to move by tunneling throughout the energy barriers which are developed at the interfaces between different metallic regions of the sample. In the case of Ni nanoparticles deposited on the CNT fiber surface a tunnel mechanism can be settled once the $\mathrm{Ni}$ nanoparticles are very close or the energy barriers at their interfaces are low enough.

The energy barrier $U_{0}$ can be calculated by the knowledge of the parameters $T_{1}$ and $T_{2}$. Taking into account that the Ni nanoparticles have dimensions $d=115 \mathrm{~nm}$ and $d=140 \mathrm{~nm}$ for 115 and $140 \mathrm{~nm}$ samples, respectively, we can assume

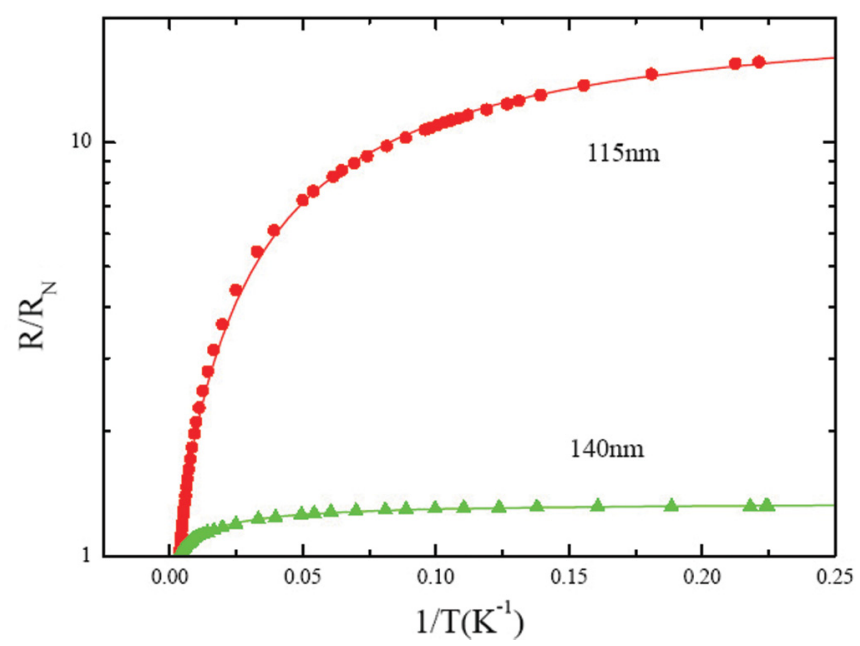

FIG. 6. (Color online) Normalized resistance as a function of $1 / T$ of the same samples reported in Fig. 5. The lines are fit to the data following the FIT model. $w=5 \mathrm{~nm}$ for the $115 \mathrm{~nm}$ sample and, as a consequence, $w=4 \mathrm{~nm}$ for the $140 \mathrm{~nm}$ sample. The values of $U_{0}$ calculated by the $T_{1} / T_{2}$ ratios are 6 and $0.2 \mathrm{meV}$ for 115 and $140 \mathrm{~nm}$ samples, respectively. This reduction in the energy barriers explains the reduction in the electrical resistance of the $140 \mathrm{~nm}$ sample with respect to the semiconducting $115 \mathrm{~nm}$. Because the thermal energy at the lowest measured temperature $(T=5 \mathrm{~K})$ is $k_{B} T=0.4 \mathrm{meV}$, the effect of $U_{0}$ on the transport properties is much more marked for the $115 \mathrm{~nm}$ sample than in the $140 \mathrm{~nm}$. The presence of the barrier energy in the latter sample is smeared out by the thermal effects and a metallic behavior appears much more possible. This is confirmed by the positive value of the temperature coefficient of the resistance for this sample below $5 \mathrm{~K}$ as shown in the inset of Fig. 2 .

The existence of a progressive transition from the semiconducting behavior exhibited by the $45 \mathrm{~nm}$ sample to the metallic one displayed by the $200 \mathrm{~nm}$ sample finds convincing evidence in the $R$ vs $T$ data of the $150 \mathrm{~nm}$ sample reported in Fig. 7. As already commented in Fig. 2, a metallic-insulating (M-I) transition appears in this sample at $T=34 \mathrm{~K}$. The dashed line is a fit to the data following the FIT model for $T<34 \mathrm{~K}$, whereas the solid line is a fit to the data using the BG model corrected for magnetic effects for $T>34 \mathrm{~K}$. The parameters obtained for the metallic part of the curve are in agreement with that expected in the case of metallic ferromagnetic conductor. The parameters obtained for the FIT model were $T_{1}=0.23 \mathrm{~K}$ and $T_{2}=6.6 \mathrm{~K}$. Assuming a distance between Ni nanoparticles of $w=1 \mathrm{~nm}$ yields a potential barrier $U_{0}=16 \mu \mathrm{eV}$. This value for $U_{0}$ is considerably lower than the thermal energy and therefore the presence of an energy barrier cannot be ascribed to the cause of the emerging semiconducting behavior below $34 \mathrm{~K}$. Moreover, the attempt to fit the low temperature part with the VRH expression returns unphysical values of the $T_{M}$ parameter $\left(T_{M}<10^{-5} \mathrm{~K}\right)$ which also rules out this mechanism of conduction.

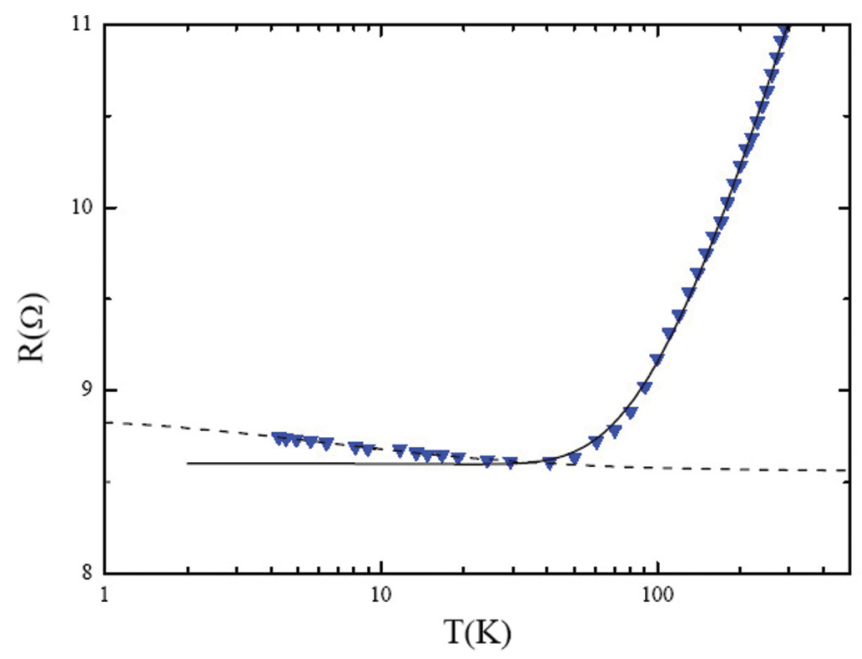

FIG. 7. (Color online) Temperature dependence of the resistance for the $150 \mathrm{~nm}$ sample. The dashed line is a fit to the data following the FIT model. The solid line is a fit to the data following the BG model corrected with the magnetic interaction. The intersection between the curves is at $T=34 \mathrm{~K}$ which is the metal insulator transition temperature. 
The mechanism for the upturn in the resistance at low temperature cannot be accounted for by the FIT and VRH model. Very likely in this case a localization mechanism should be considered in order to provide a satisfactory explanation, a conjecture which is consistent with the fact that the electron hopping length decreases with the temperature. Adopting the values of $\xi$ calculated for the $45 \mathrm{~nm}$ sample, we obtain at the M-I transition temperature $T_{\mathrm{M}-\mathrm{I}}=34 \mathrm{~K}$ an hopping length $r=150 \mathrm{~nm}$ which is in excellent agreement with the measured grains dimension in the $150 \mathrm{~nm}$ sample. Supported by these indications, we can deduce that the observed upturn in the $R$ vs $T$ data below $T=34 \mathrm{~K}$ for the $150 \mathrm{~nm}$ sample could indeed be ascribed to the localization of electrons inside each grain. When $T<T_{\mathrm{M}-\mathrm{I}}, r>d$, that is, the hopping mechanism works and the semiconducting behavior appears. For $T>$ $T_{\mathrm{M}-\mathrm{I}}, r<d$, that is, hopping is not possible and the electrons move along some percolative path formed between the voltage electrodes, yielding metallic conduction.

\section{CONCLUSIONS}

We have performed a systematic analysis of the transport mechanism in one-dimensional granular structures of nickel nanoparticles coating carbon nanotube fibers. Our experimental results show how the electrical conduction in the nickel granular system is affected by grain dimension, external fields, and temperature. We have shown evidence of a metal-insulator transition whose existence can be attributed to the reduction of the distance between the grains. The grain distance also gives rise to different conduction regimes generated by the variations in the charging energy and thermal fluctuations and the metal-insulator transition detected in one sample suggests that localization effects are present inside the grains. We believe that our analysis opens interesting perspectives from the applied physics point of view in the search for new materials serving in interconnect technology. The thermal conductivity of the bare fibers that we have used is of the order of $20 \mathrm{~W} / \mathrm{m} \mathrm{K}$, at room temperature: ${ }^{15}$ It is likely that increasing the density of the nanotubes inside the fibers the thermal conductivity could also be improved to get in the range of hundreds of $\mathrm{W} / \mathrm{m} \mathrm{K}$. These values would be comparable with those of several metals and semiconductors and therefore coated nanotube fibers could serve several purposes in the field of interconnections, provided that a systematic analysis of the structural and electrical properties of the coating effect shall be available for understanding their limits and potentialities.

\section{ACKNOWLEDGMENTS}

The work has been supported by the GESTO program of the Regione Lazio (Italy), the Welch foundation (C-1668), and AFOSR (FA9550-09-1-0590).
*Also CNR-SPIN Institute, Italy.

${ }^{1}$ I. S. Beloborodov, A. V. Lopatin, and V. M. Vinokur, Rev. Mod. Phys. 79, 469 (2007).

${ }^{2}$ I. S. Beloborodov, K. B. Efetov, A. V. Lopatin, and V. M. Vinokur, Phys. Rev. Lett. 91, 246801 (2003).

${ }^{3}$ T. B. Tran, I. S. Beloborodov, J. Hu, X. M. Lin, T. F. Rosenbaum, and H. M. Jaeger, Phys. Rev. B 78, 075437 (2008); T. B. Tran, I. S. Beloborodov, X. M. Lin, V. M. Vinokur, and H. M. Jaeger, Phys. Rev. Lett. 95, 076806 (2005).

${ }^{4}$ P. Sheng, B. Abeles, and Y. Arie, Phys. Rev. Lett. 31, 44 (1973).

${ }^{5}$ N. F. Mott and E. A. Davis, Electronic Processes in Non-Crystalline Materials (Clarendon, Oxford, 1979); B. I. Shklovskii and A. L. Efros, Electronic Properties of Doped Semiconductors (Springer, Berlin, 1984).

${ }^{6}$ I. S. Beloborodov, A. Glatz, and V. M. Vinokur, Phys. Rev. Lett. 99, 066602 (2007).

${ }^{7}$ K. B. Efetov and A. Tschersich, Europhys. Lett. 59, 114 (2002).

${ }^{8}$ R. Sachser, F. Porrati, C. H. Schwalb, and M. Huth, Phys. Rev. Lett. 107, 206803 (2011).

${ }^{9}$ P. Sheng, Phys. Rev. B 21, 2180 (1980).

${ }^{10}$ R. Saito, G. Dresselhaus, and M. S. Dresselhaus, Physical Properties of Carbon Nanotubes (Imperial College Press, London, 1998).
${ }^{11}$ Handbook of Chemistry and Physics (CRC, Boca Raton, FL, 2005).

${ }^{12}$ A. J. Blodgett and W. E. Spicer, Phys. Rev. Lett. 15, 29 (1965).

${ }^{13}$ L. M. Ericson et al., Science 305, 1447 (2004).

${ }^{14}$ E. Tamburri, F. Toschi, V. Guglielmotti, E. Scatena, S. Orlanducci, and M. L. Terranova, J. Nanopart. Res. 11, 1311 (2009); S. Orlanducci, E. Tamburri, V. Guglielmotti, F. Toschi, M. L. Terranova, M. Lucci, I. Ottaviani, M. Salvato, and M. Cirillo, IEEE Trans. Nanotech. 10, 764 (2011).

${ }^{15}$ W. Zhou, J. Vavro, C. Guthy, K. I. Winey, J. E. Fischer, L. M. Ericson, S. Ramesh, R. Saini, V. A. Davis, C. Kittrell, M. Pasquali, R. H. Hauge, and R. E. Smalley, J. Appl. Phys. 95, 649 (2004).

${ }^{16}$ M. Salvato, M. Lucci, I. Ottaviani, M. Cirillo, E. Tamburri, I. Cianchetta, V. Guglielmotti, S. Orlanducci, M. L. Terranova, and M. Pasquali, Phys. Rev. B 84, 233406 (2011).

${ }^{17}$ J. M. Ziman, Electrons and Phonons (Clarendon, Oxford, 1960).

${ }^{18}$ I. Mannari, Prog. Theor. Phys. 22, 335 (1959).

${ }^{19}$ H. E. Romero and M. Drndic, Phys. Rev. Lett. 95, 156801 (2005); D. Yu, C. Wang, B. L. Wehremberg, and P. Guyot-Sionnest, ibid. 92, 216802 (2004); R. Parthasarathy, X. M. Lin, K. Elteto, T. F. Rosenbaum, and H. M. Jaeger, ibid. 92, 076801 (2004). 